\title{
The Inverse Mean Field Method and the Energy Dependence of the Nuclear Potential
}

\author{
E. F. Hefter* and K. A. Gridnev** \\ * Instituto de Fisica, Universidad Nacional Autónoma de México, México D. F., México \\ and \\ Institut für Theoretische Physik, Universität Hannover, Appelstraße 2, D-3000 Hannover $1^{\text {a }}$. \\ ** NIIF, Leningrad State University, Leningrad, USSR.
}

Z. Naturforsch. 38a,813-820 (1983); received April 11, 1983

\begin{abstract}
The foundations of the inverse mean field method (Imefim) and its relation to traditional approaches are discussed. Imefim predicts the energy dependence of the real central (nuclear) part of the optical model potential to have the functional form $\left(1+e E_{\mathrm{p}}\right)^{-3}$. Treating for the time being the constant $e$ as an adjustable parameter, this prediction is shown to compare nicely with well-established formal and heuristic results.
\end{abstract}

\section{Introduction}

The aim of this note is to draw attention to the energy dependence of the optical model as predicted by a rather unconventional approach to nuclear physics, which has been termed the inverse mean field method or in short Imefim [1-7]. Similarly as e.g. time-dependent Hartree-Fock (TDHF), Imefim starts off with the mean field picture reducing the full many-body Schrödinger equation to the single-particle Schrödinger problem with the mean field $U$ (this explains the "mefi" in Imefim).

Traditionally it is attempted to solve Schrödingertype problems via "direct" methods; i.e. (due to the historical experience gained in atomic physics, where the (Coulomb) potential is known to a high precision) one begins with an assumed or given knowledge of the macroscopic potential, $U$, or of the interparticle interaction, $V_{\mathrm{nn}}$, to evaluate wavefunctions, energy eigenvalues and all further quantities of interest. However, in nuclear physics our knowledge of $U$ and $V_{\mathrm{nn}}$ is not (yet?) a sufficiently good one. Hence, it has been suggested to employ "inverse" methods (for inverse methods in general cf. e.g. [8]) for attacking the problem (this explains the "I" in Imefim). The required input is then given by the complete energy spectrum of the Schrödinger

\section{a Permanent address.}

Reprint requests Dr. E. F. Hefter, Institut für Theoretische Physik, Universität Hannover, Appelstraße 2, D-3000 Hannover 1. operator, i.e. the $\mathrm{N}$ bound-state energies, $E_{\mathrm{n}}$, plus the (infinite set of) reflection coefficients, $R\left(E_{\mathrm{p}}\right)$ with $E_{\mathrm{p}}>0$, containing the information on the continuous part of the spectrum. (The symbol $E_{\mathrm{p}}$ is used to denote the kinetic energy of the incident nucleon in nucleon-nucleus scattering which we consider in this paper.) In principle, all the input data could be obtained by direct experimental measurements to allow then for the evaluation of the auxiliary potential, $U$, and the wavefunctions. Then one could proceed (in the same way as in the case of the direct method) to extract further quantities of interest like e.g. the nuclear densities.

Below we do not want to elaborate on the static shell model potential, $U$, but rather on the real central (nuclear) part, $V$, of the optical model interaction, $V_{\mathrm{om}}$, employed in the description of elastic nucleon scattering data.

$$
V_{\mathrm{om}}=V_{\mathrm{Cl}}+V+i \cdot W+V_{\mathrm{so}} .
$$

$V_{\mathrm{Cl}}, W$ and $V_{\text {so }}$ correspond to the Coulomb potential, the imaginary contributions (volume and surface) and the spin-orbit interaction, respectively. We will only be concerned with $V$.

Experience suggests to treat the depth of the potential $V$ as a function of the projectile energy, $E_{\mathrm{p}}$. However, neither formal arguments nor phenomenological studies were so far able to provide us with reliable and unique overall predictions for the appropriate energy dependence $V\left(E_{\mathrm{p}}\right)$. In a recent heuristic discussion [9] the form

$$
V\left(E_{\mathrm{p}}\right)=-\left(V_{0}-\alpha E_{\mathrm{p}}+\beta E_{\mathrm{p}}^{2}\right)
$$

0340-4811 / $83 / 0800-0813 \$ 01.30 / 0$. - Please order a reprint rather than making your own copy. 
with the adjustable parameters $\alpha$ and $\beta$ has been shown to yield a closer correspondence to experiment, than other phenomenological dependences of an exponential type like e.g.

$$
V\left(E_{\mathrm{p}}\right)=-52.6 \exp \left(-0.0066 E_{\mathrm{p}}\right)
$$

[10]. Moreover, it has been indicated that the $V\left(E_{\mathrm{p}}\right)$ of (2) is not just appropriate for positive, but also for negative energies (bound-states) implying that the real central part of the shell-model potential, $U$, coincides for $E_{\mathrm{p}}=0 \mathrm{MeV}$ with $V\left(E_{\mathrm{p}}\right)$, i.e. $V\left(E_{\mathrm{p}}=0\right)=U$ (cf. [9] and references).

The main message we would like to convey in this note is that Imefim yields in contrast to other approaches a definite prediction for the appropriate energy dependence. If this energy dependence can be shown to be in line with empirical results and the notions of an effective mass and of an energy dependent mass operator, then there would be further arguments for intensified studies into Imefim's mathematical structure and for its application to nuclear physics.

The paper is structured in such a way that we recollect in Section 2, the basic formulae of Imefim, its interrelation with traditional approaches and the functional form of its projectile energy dependence (originally derived in the context of fluid dynamical problems!). In Sect. 3 it is discussed how the energy dependence of Imefim compares with phenomenological results and to what extent it is consistent with the ones of traditional approaches. The last part is devoted to a short summary.

\section{The Inverse Mean Field Method (Imefim)}

\subsection{Basic formulae of Imefim}

The equation of motion that should be solved in a nonrelativistic quantum mechanical many-body problem like the one posed by the atomic nucleus, is the time-dependent Schrödinger equation

$$
H(\boldsymbol{r}, t) \Psi(\boldsymbol{r}, t)=i \hbar \partial_{t} \Psi(\boldsymbol{r}, t) .
$$

(We use $\partial_{t}, \partial_{x x}$, etc. to denote partial differentiation with respect to the variables $t$ and $x$, resp.) The physically motivated mean field picture reduces this many-body equation to a set of $N$ single-particle equations with the mean field $U$ and the $N$ statevectors $\psi_{n}(\boldsymbol{r}, t)$. Let us simplify the problem still further by considering only spherically symmetric (three-dimensional) problems, i.e. we exclude deformed nuclei from our considerations. The substitutions

$$
\begin{aligned}
& x \rightarrow r \text { and } \\
& U(x, t) \rightarrow U(r, t)+\hbar^{2} l(l+1) / 2 m r^{2}
\end{aligned}
$$

together with the appropriate boundary conditions $\left(\psi_{n}(x=0)=0\right)$ reduce the equations to be solved to

$$
\begin{aligned}
& \quad-M \partial_{x x} \psi_{n}(x, t)+U(x, t) \psi_{n}=i \hbar \partial_{t} \psi_{n}(x, t) \\
& n=1,2, \ldots, N
\end{aligned}
$$

with

$$
\begin{aligned}
& M=: \hbar^{2} / 2 m \\
& (m \text { - reduced mass of the system) } .
\end{aligned}
$$

Instead of attacking the problem as usual via direct methods, we apply inverse methods/look for an evolution equation in $U(x, t)$. This lead us eventually to the conclusion (in view of the material which appeared to-date in print, the expression "working hypothesis" might be more appropriate) that the $N$ Eqs. (5) are equivalent to the system of coupled equations:

$$
\begin{aligned}
-M \partial_{x x} \psi_{n}(x, t)+U(x, t) \psi_{n}=E_{n} \psi_{n} ; \\
n=1,2, \ldots, N \\
\partial_{t} U(x, t)=v_{0} \partial_{x} U+\left[6 U \partial_{x} U-M \partial_{x x x} U\right] \\
\quad+D(x, t) \partial_{x x} U+f\left(E_{0}, e^{\prime} E_{\mathrm{p}}\right) U .
\end{aligned}
$$

(As a matter of fact, (8) just yields an $l=0$ equivalent potential; for each $l \neq 0$ a further more complicated equation of the same structure as (8) arises. Another point is that the operator $\partial_{t}$ in (8) should actually be replaced by a slightly different one $[2,4]$. However, for the subsequent discussion these two points are not substantial so that we suppress them.) Equations (7), (8) are both Galilean invariant so that one may use with no loss of generality $v_{0}=0$ for the speed of the system as a whole. The function $D(x, t)$ in $(8)$ is only included for the sake of completeness, i.e. we are going to use $D=0$ throughout this paper, so that we avoid a detailed discussion of the physics contained in $D$. It is simply stated that $D$ describes dissipative effects that are important for the description of inelastic processes. We did not yet provide a microscopic derivation of the function $f\left(E_{0}, e^{\prime} E_{\mathrm{p}}\right)$. It should at present only be borne in mind that $E_{0}$ has to be associated with the binding energy of the system/ 
nucleus consideration. Below we absorb for the time being $E_{0}$ and the constant $e^{\prime}$ into a single coefficient $e$, which is treated as an adjustable parameter, cf. Equation (13). In work that is still in progress this arbitrariness in $e, e^{\prime}$ and $E_{0}$ is to be removed by a microscopic derivation of these quantities (yet, the time scale required for this enterprise may be fairly large).

\subsection{The conservative version of Imefim \\ (i.e. $D=0=f$ )}

The general solution of (8) has to be obtained numerically, but its conservative version,

$$
\partial_{t} U(x, t)=6 U \partial_{x} U-M \partial_{x x x} U,
$$

has under certain circumstances analytical solutions. The point is that $\left(8^{\prime}\right)$ requires a knowledge of the complete spectrum of the related Schrödinger equations, (7). Then application of inverse scattering methods (via the Gelfand-Levitan-Marchenko equation) allows for a numerical solution of $\left(8^{\prime}\right)$ $[2,8]$. It turned out to be a real surprise that one obtains for reflectionless potentials, i.e. for $R(k)=$ $R\left(E_{\mathrm{p}}\right)=0$ for all $E_{\mathrm{p}}>0$, an analytical solution to $\left(8^{\prime}\right)$. For $N$ such reflectionless potentials that home $N$ energy eigenvalues the solution of $\left(8^{\prime}\right)$ reads

$$
\begin{aligned}
U_{N}(x, t) & =\sum_{i=1}^{N} U_{N i}(x, t)=\sum_{i}-4 M K_{i} \varrho_{i}(x, t) \\
& =\sum_{i}-4 M K_{i} \psi_{i}^{2}(x, t)=-2 M \partial_{x x} \ln (\operatorname{det} F)
\end{aligned}
$$

with $F_{i j}=\delta_{i j}+\sqrt{f_{i} f_{j}} /\left(\sqrt{E_{i}}+\sqrt{E_{j}}\right)$,

$$
f_{i}(x, t)=2 \sqrt{E_{i}} \exp \left(2 K_{i}\left(x-x_{0 i}-4 E_{i} L_{i} t\right)\right) \text {; }
$$

$$
L_{i}=\text { const ; }
$$

$K_{i}^{2}=:-2 m E_{i} / \hbar^{2}=-E_{i} / M ; \quad E_{i}<0$.

From (9) it is seen that the potential, $U_{N}$, is a (nonlinear) sum of the densities $\varrho_{i}$. The boundary conditions (cf. below (4)) enforce the use of $x_{0 i}=0$ for all $i$. The constants $L_{i}$ are fixed by other demands (or eliminated if we use $t=0$ ) so that they are no more at our disposal. Hence, the $N$-energy eigenvalue $/ N$-reflectionless potential $/ N$-soliton solution, $U_{N}$, of $\left(8^{\prime}\right)$ is uniquely determined by the $N$ boundstate energy eigenvalues $[2,8,11-14]$. It turned out that for our purposes $U \cong U_{N}$, i.e. $R(k)=0$, is indeed a very good approximation $[2,14]$ so that we consider below only this rather simple case.
For $N=1$ Eq. (9) yields the following expressions for the potential $U_{1}$ and for the wavefunction $\psi_{1}$ which it homes:

$$
\begin{aligned}
& U_{1}(x, t)=-U_{01} \cdot \operatorname{sech}^{2}\left(K_{1}\left(x-x_{01}-4 E_{1} L_{1} t\right)\right) \\
&=-4 M K_{1} \psi_{1}^{2}(x, t) \\
& \psi_{1}(x, t)=\sqrt{K_{1} / 2} \cdot \operatorname{sech}\left(K_{1}\left(x-x_{01}-4 E_{1} L_{1} t\right)\right) ; \\
& U_{01}=\hbar^{2} K_{1}^{2} / m
\end{aligned}
$$

Further below we will come back to the expression for $U_{1}(x, t)$ at $t=0$.

Let us now take the solution of $\left(8^{\prime}\right)$, i.e. the $U_{N}$ of (9), and insert it into the Schrödinger equation (7). What we obtain is a nonlinear version of the Schrödinger equation:

$$
\begin{gathered}
-M \partial_{x x} \psi_{n}(x, t)+\left[-4 M \sum_{i=1}^{N} K_{i} \psi_{i}^{2}(x, t)\right] \psi_{n}=E_{n} \psi_{n} ; \\
n=1,2, \ldots, N .
\end{gathered}
$$

Defining a one-column vector $\Psi(x, t)$ with the $N$ elements $\sqrt{4 M K_{i} / V_{0}} \psi_{n}$ the $N$ equations (11) may rewritten in the compact form

$$
\begin{array}{r}
-M \partial_{x x} \Psi(x, t)-V_{0} \Psi^{2} \Psi=\hat{E} \Psi ; \\
\hat{E}=:\left(E_{1}, E_{2}, \ldots, E_{N}\right)
\end{array}
$$

[7]. Equation $\left(11^{\prime}\right)$ is just the multicomponent version of the famous non-linear Schrödinger equation extensively discussed and applied in various branches of physics ranging from hydrodynamics over plasma physics to nonlinear optics [15].

\subsection{Imefim and conventional approaches}

A detailed discussion of the interrelation of Imefim with traditional approaches would exceed the frame of this contribution so that we limit ourselves to a few words related to the structures of the equations used in some approaches. To start with, (11') has the same functional form as semi-phenomenological nonlinear Schrödinger equations that arise when fluid dynamical considerations/models are applied to nuclear physics, cf. e.g. [16] and the references given therein. This point gives some additional weight to the notion that Imefim might indeed provide a useful tool for discussing nuclear physics.

However, in the context of (1-d) studies into the (time-dependent) Hartree Fock method it has been shown that (11) arise if one assumes an interparticle 
force of the Delta-type [17, 18]. Hence, we are again on common grounds with well established and successful approaches. But it should be borne in mind that within TDHF these equations arise due to a drastic simplification of the interparticle forces; Imefim yields them on more general grounds. Therefore the mean field theory implied by Imefim is more general than the one implied by the corresponding (TD)HF equations [5]. Furthermore, (11) have recently been derived and applied to a discusssion of the interrelations between TDHF and the time-dependent mean field S-matrix approach [19].

The arising notion that Imefim is indeed intimately interrelated with conventional approaches is to be discussed elsewhere in more detail.

\subsection{Imefim and nuclear structure problems (i.e. $D=0=f$ )}

If it is desired to discuss static nuclear structure problems, then we have obviously to use $D=0=f$. Any other value for $D$ or $f$ would imply that the energy eigenvalues of the related Schrödinger equations change with time. Limiting ourselves to the ground-state properties this would obviously be a highly undesirable feature. Hence, we have to use $\left(8^{\prime}\right)$ instead of the complete version (8).

As indicated in Part 2.2, the $N$ bound-state energy eigenvalues $E_{n}$ are the only external input we need for a unique specification of $U_{N}$ and the related wavefunctions, densities, etc. However, if we do not restrict ourselves to the simple single-particle picture, then we have to multiply the resp. densities by the occupation numbers/spectroscopic factors taken from analysis of experimental data. But in any case we have a well defined prescription for the input to be used. Preliminary calculations indicate e.g. that such calculations within Imefim reproduce/ predict the odd-even staggering in the nuclear rms radii (e.g. of the $\mathrm{Ca}$ isotopes). - To our knowledge there is so far no other consistent/satisfactory explanation and interpretation of such data.

Even without explicit calculations, it is from the preceeding subsection obvious that Imefim should lead to similar results as e.g. (TD)HF calculations. From this sub-section we would like to take the notion that Imefim is seemingly a useful tool for nuclear structure calculations, a point to be resumed in forthcoming contributions.

\subsection{The energy dependence of Imefim}

(i.e. $D=0$ in (8))

In the analysis of elastic scattering experiments the optical model enjoys considerable success. It reduces the problem to potential scattering, i.e. one has to solve a Schrödinger eigenvalue problem with a positive energy (the kinetic energy of the incident projectile) and the potential $U_{\text {om }}$, cf. (1). As indicated in the introduction, the contribution $V$ to $V_{\text {om }}$ has for the kinetic energy $E_{\mathrm{p}}=0$ of the projectile to be identified with the shell-model potential $U$. Within Imefim that implies that $V\left(E_{\mathrm{p}}=0\right)$ has to correspond to $U=U_{\mathrm{N}}$. The dependence of $V\left(E_{\mathrm{p}}\right)=U_{\mathrm{N}} g\left(E_{\mathrm{p}}\right)$ is produced by (8) with $D=0$ and with $f\left(E_{0}, e^{\prime} E_{\mathrm{p}}\right) \neq 0$. The significance of $f\left(E_{0}, E_{\mathrm{p}}\right)$ is made more transparent if we rewrite it in the form $f\left(E_{0}, E_{\mathrm{p}}\right)=f^{\prime}\left(E_{0}, E_{\mathrm{p}}\right) \delta(t)$. The Deltafunction stresses the point that the function $f$ just helps to set the initial conditions; i.e. in exact analogy to experiment where the accelerator, etc. are adjusted so as to produce a constant beam with a fixed intensity and kinetic energy. After these manipulations the incident beam is accurately defined and its specifications do (in the ideal case at least) not change with time. In a very similar fashion the function $f\left(E_{0}, E_{\mathrm{p}}\right)$ describes the changes to be performed when going over from one projectile energy to another one [2].

The influence of $f\left(E_{\mathrm{p}}\right)$ on the initial amplitude of a single soliton or an $\mathrm{N}$-soliton solution (at $E_{\mathrm{p}}=0$ ) is to reduce its amplitude with increasing $E_{\mathrm{p}}$. Since the overall results corresponds (neglecting surface effects) to a simple scaling of the soliton, it is all the same whether we study this effect on a $\mathrm{N}$-soliton solution or on a single soliton. Hence, we simplify life by considering an "average" soliton $\bar{U}$ with the amplitude $\bar{U}_{0}$

$$
\begin{gathered}
\bar{U}\left(x, E_{\mathrm{p}}\right)=\bar{U}_{1}\left(x, E_{\mathrm{p}}\right)=-\bar{U}_{0}\left(E_{\mathrm{p}}\right) \cdot \operatorname{sech}^{2}(\bar{K} \cdot x) ; \\
\bar{U}_{0}=\hbar^{2} \bar{K}^{2} / m .
\end{gathered}
$$

$\bar{U}_{0}$ is to be normalized to the average depth of the (shell-model) potential $U_{\mathrm{N}}$ of (9) or to empirical potentials. $\bar{U}$ and its volume integral are (in one and in three dimensions) uniquely specified by the average amplitude $\bar{U}_{0}$ and the reduced mass $m$.

The effect of the function $f\left(E_{0}, e^{\prime} E_{\mathrm{p}}\right)$ on the soliton's amplitude has originally been studied in the context of fluid dynamics where the quantities involved have naturally different physical inter- 
pretations. Applying the results of these studies [20] to our problem, the $E_{\mathrm{p}}$-dependence of the amplitude $\bar{U}_{0}$ reads

$$
\bar{U}_{0}\left(E_{\mathrm{p}}\right)=\bar{U}_{0}\left(E_{\mathrm{p}}=0\right)\left(1+e E_{\mathrm{p}}\right)^{-3}
$$

with $e=: e^{\prime} /\left(1+E_{0}\right)$.

As mentioned above, we absorbed the two (so far unspecified) constants $e^{\prime}$ and $E_{0}$ into the single constant $e$, which we treat for the time being as a fit parameter. (Before attempting the rather laborious derivation of these constants from first principles.) In the next section we would like to discuss to what extent the energy dependence of (13) is a realistic one as far as nuclear physics problems are concerned.

But before proceeding to such a comparison, let us have a second look at (12) and (13). Considering the definition of $\bar{U}_{0}$, it is obviously at first sight not clear whether the energy dependence should be attributed to the average wavenumber $\bar{K}$ or to the mass $m$. But in view of the point that $\bar{K}$ is related to the energy eigenvalues of the nuclei which are elastically scattered, it has to be required that the energy eigenvalues and hence $\bar{K}$ have to be the same for large times before and after the collision event. Hence, the mass $m$ is left as the only quantity that contains an explicit energy dependence:

$$
m^{*}=: m\left(E_{\mathrm{p}}\right)=m\left(E_{\mathrm{p}}=0\right)\left(1+e E_{\mathrm{p}}\right)^{3} .
$$

In such a manner Imefim predicts a definite energy dependence for the potential $\bar{U}$ (or $U_{\mathrm{N}}$ ) or for the emerging "effective" mass $m^{*}$. If this energy dependence does not yield reasonable results, then we have strong indications that there is something wrong with Imefim or at least with its energy dependence. A positive outcome would certainly encourage further studies and indicate that it is sensible to proceed towards a microscopic derivation of this energy dependence/the constants involved.

\section{The Energy Dependence of the Nuclear Potential}

In this section we would like to compare some phenomenological energy dependences of the real central (nuclear) part of the optical model interaction, i.e. of $V\left(E_{\mathrm{p}}\right)$, with the energy dependence predicted by Imefim, cf. (13). Let us first have a

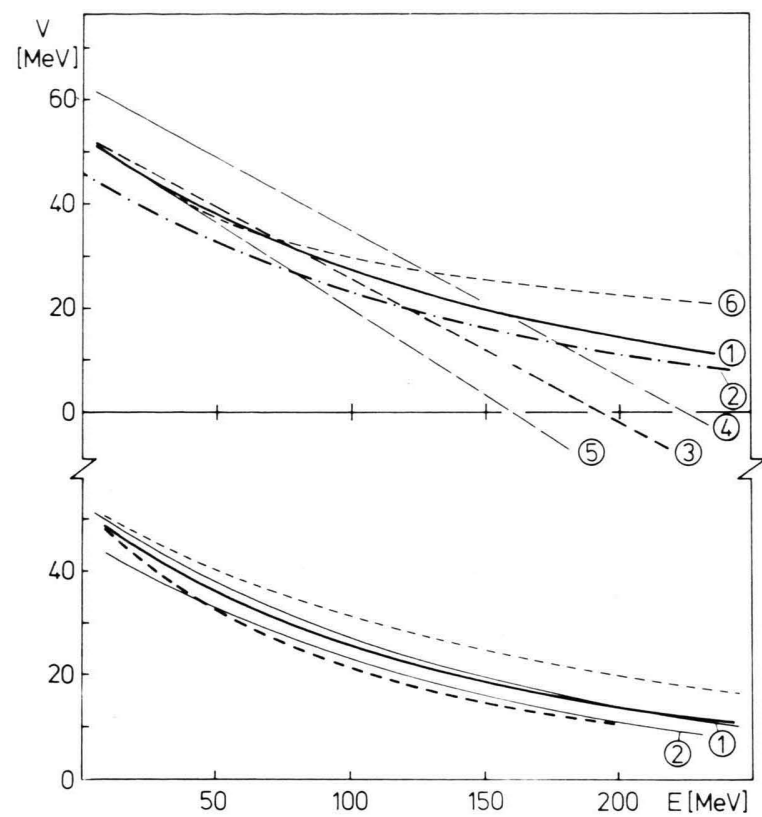

Fig. 1. The depth $U=V\left(E_{\mathrm{p}}\right)$ of the real (nuclear) part of the optical model potential for elastic nucleon-nucleus scattering is plotted versus the projectile energy $E=E_{\mathrm{p}}$. For a discussion of curves (1) to (6) cf. the text. Thin broken, bold full and bold broken curves in the lower part are based on (13) with $e=0.0019,0.0027$ and 0.0035 , respectively, cf. also the text.

look at various phenomenological energy dependences as extracted/proposed by different groups:

In the upper half of Fig. 1, the curve labelled (5) stems from the contribution of Rosen et al. [21] and it is supposed to be valid for projectile energies from $5 \mathrm{MeV}$ to $24 \mathrm{MeV}$. An extrapolation procedure employed by Butler et al. [22] extends it towards energies beyond $24 \mathrm{MeV}$, cf. curve (6). The energy dependence due to Kidwai and Rook [23], cf. curve (4), should be appropriate for kinetic energies of the incident nucleons up to $50 \mathrm{MeV}$ while curve (3), cf. can Oers and Lerner et al. [24], covers the energy range from 10 to $180 \mathrm{MeV}$. The $V\left(E_{\mathrm{p}}\right)$ suggested by Engelbrecht and Fiedeldey, curve (2), - which later on has been subjected to slight corrections (by Eder et al.), cf. curve (1) - is said to be suitable for energies up to about $180 \mathrm{MeV}$ [10].

The information contained in these curves can at best be considered to be a qualitative one. The reasons for this statement are fairly obvious: According to (1), $V$ is just one out of several terms contributing to the total optical model potential. It is not just $V\left(E_{\mathrm{p}}\right)$, but the interference between the 
different potential terms which generates the final data to be compared to experiment. And in general different groups use different parametrizations/ geometries for the various terms. Hence, a truly detailed comparison would have to keep track of all these features. In general that is not done and one is content to discuss only the energy dependence of the depth of the potential $V\left(E_{\mathrm{p}}\right)$. Below we follow this (mis-)usage. But trying to be at least a bit more careful and attempting to include at least some of the finer details in our considerations, we arrived in our study of these potentials eventually at the (nevertheless rather personal) decision to take curves (1), (2) as the relevant data to be compared to the predictions of Imefim. (A choice which has to do some injustice to the other potentials, which are by no means inadequate for the purposes they have been tailored for.) In addition to these data we refer also to some further work of a similar type, which contains phenomenological data and results based on dispersion relations [25]. Inclusion of the energy dependence(s) of Bauer et al. [9] which are based on a rather detailed numerical study will be shown to support the trends observed in the numerics involving (13).

In the lower part of Fig. 1 curves (1) and (2) are repeated. As indicated in Sect. 2, we normalize the $\bar{U}_{0}\left(E_{\mathrm{p}}=0\right)$ of (13) to the empirical data (of [10], cf. also (3); i.e. $\left.\bar{U}_{0}\left(E_{\mathrm{p}}=0\right)=: V\left(E_{\mathrm{p}}=0\right)=52.6 \mathrm{MeV}\right)$.

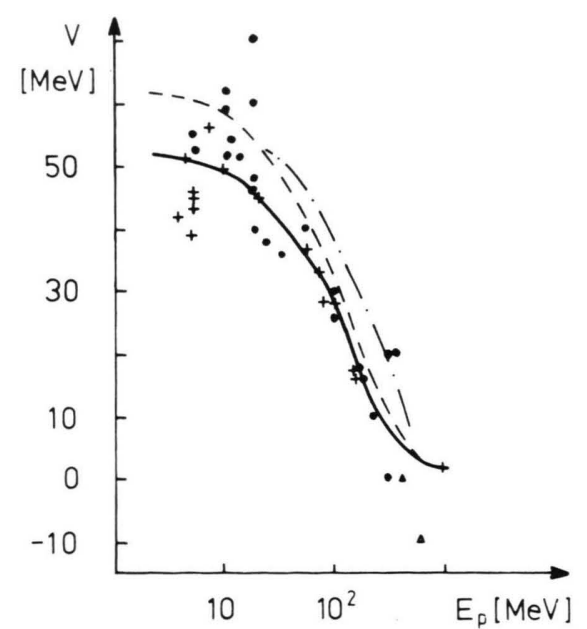

Fig. 2. The depth $V$ is plotted as a function of $E_{\text {p. Dots }}$ and crosses correspond to phenomenological results/fits; dotted curve - based on dispersion relations [25]; broken and full curves are due to the soliton approach with different normalizations and with $e=0.0027$.
Table 1. The (magnitudes of the) parameters $\alpha$ and $\beta$ of Bauer et al. [9], cf. Eq. (2), are displayed for ${ }^{40} \mathrm{Ca}$ and "all" (i.e. ${ }^{40} \mathrm{Ca},{ }^{58} \mathrm{Ni},{ }^{90} \mathrm{Zr}$ and ${ }^{208} \mathrm{~Pb}$ ) and put in relation to the ones due to Imefim, cf. (15) and (16).

\begin{tabular}{|c|c|c|c|}
\hline & $\alpha$ & $\beta$ & $\delta=10 e^{3} \bar{U}_{0}$ \\
\hline $\begin{array}{l}{ }^{40} \mathrm{Ca} \text { with } \\
U_{0}=56.3 \mathrm{MeV}\end{array}$ & $\begin{array}{r}0.41 \\
\pm .06\end{array}$ & $\begin{array}{r}0.0009 \\
\pm .0003\end{array}$ & \\
\hline $\begin{array}{l}\text { "all" with } \\
U_{0}=52.4 \mathrm{MeV}\end{array}$ & $\begin{array}{r}0.37 \\
\pm .02\end{array}$ & $\begin{array}{r}0.0007 \\
\pm .0001\end{array}$ & \\
\hline $\begin{array}{cc} & =0.0019 \\
\text { Imefim } & \mathbf{0 . 0 0 2 7} \\
& 0.0035\end{array}$ & $\begin{array}{l}0.30 \\
\mathbf{0 . 4 2} \\
0.55\end{array}$ & $\begin{array}{l}0.0011 \\
\mathbf{0 . 0 0 2 3} \\
0.0039\end{array}$ & $\begin{array}{l}0.000009 \\
\mathbf{0 . 0 0 0 0 1 0} \\
0.000023\end{array}$ \\
\hline
\end{tabular}

The constant $e$ appearing in (13) is for the time being varied to accomplish a good agreement between the results of Imefim and the empirical curve(s). For the value $e=0.0027$ (bold full curve) the correspondence between them is indeed a very close one. In view of the deviations between the different phenomenological curves displayed in the upper half of the figure it is even excellent. The broken curves illustrate the extent to which the soliton results depend on the exact value of $e$.

Dots and crosses in Fig. 2 represent empirical data copied from [25]. The dotted curve based on dispersion theory stems from the same source. Broken and full curves correspond to the predictions of Imefim; i.e. (13) with $e=0.0027$ and with $\bar{U}_{0}\left(E_{\mathrm{p}}=0\right)=61.5 \mathrm{MeV}$ and $52.5 \mathrm{MeV}$, respectively. A glance at Fig. 2 (and a look into [25]) confirm that Imefim's prediction yields an agreement with the heuristic data which is no worse than the one accomplished via dispersion theory (or other conventional approaches).

Coming back to the quadratic energy dependence of Bauer et al. [9], cf. (2), and having a second look at the functional form of $V\left(E_{\mathrm{p}}\right)$ as predicted by Imefim, (13), it is seen that there are little if any problems in reproducing/approximating (2) by aid of (13) - at least if $e$ is treated as a fit-parameter. This statement is almost self-evident if we write down the expansion for (13) (which is reasonable for $\left.e E_{\mathrm{p}} \ll 1\right)$ :

$$
\begin{aligned}
-\bar{U}_{0}\left(E_{\mathrm{p}}=0\right)\left(1+e E_{\mathrm{p}}\right)^{-3} & \\
=- & {\left[\bar{U}_{0}(0)-3 \bar{U}_{0}(0) e E_{\mathrm{p}}+6 \bar{U}_{0}(0) e^{2} E_{\mathrm{p}}^{2}\right.} \\
& \left.-10 \bar{U}_{0}(0) e^{3} E_{\mathrm{p}}^{3}+-\cdots\right] .
\end{aligned}
$$

If we terminate the expansion after the third term and compare it to (2), then the $\alpha$ and $\beta$ of (2) are 
seen to correspond to

$\alpha=3 \bar{U}_{0}\left(E_{\mathrm{p}}=0\right) e$ and $\beta=6 \bar{U}_{0}\left(E_{\mathrm{p}}=0\right) e^{2}$.

The higher order terms which are of importance at larger projectile energies appear necessarily in (15), but they have no counterpart in (2) where this effect has to be absorbed into the empirically determined constants $\alpha$ and $\beta$. In Table 1 we compare the heuristic results of [9] with the coefficients $\alpha$ and $\beta$ due to Imefim, cf. (16). Bearing in mind the (in)sensitivity of the curves in Fig. 1 to variations in $e$, the agreement of the respective $\alpha$ 's in Table 1 is simply excellent; the one in $\beta$ 's is less good. However, the fourth term in the more adequate expansion (15) would reduce the value of the $\beta$ produced by Imefim so that it would come still closer to the value of its empirical counterpart. Hence, we infer that the energy dependence of Imefim is fully in line with the one of [9], which has been thoroughly tested against a fairly large body of data.

Summarizing, the results based on the functional form of the energy dependence predicted by Imefim, cf. (13), have been shown to be consistent with the ones of phenomenological and formal approaches [9], [10, 25]. From Sect. 2.4. it is obvious that the notion of such an energy dependence is not just consistent with the traditional discussion centered around the optical model, but also with the notions of an energy dependent mass operator or of an (energy dependent) effective mass as put forward in the context of the theory of Fermi liquids and the (time-dependent) Hartree-Fock method, respectively. But the full predictive power of Imefim will only come into play after a microscopic derivation of the constant $e$ which we treated up to now as a fitparameter. Work along these lines is still at its initial stage.

\section{Summary}

We recalled the prominent features of the inverse mean field method (Imefim), drew attention to previous applications of it and to its interrelation with the (time-dependent) Hartree-Fock method and phenomenological nonlinear Schrödinger equa- tions. We then focussed our attention on the energy dependence of the depth of the real central (nuclear) part of the optical model potential, i.e. of the $V\left(E_{\mathrm{p}}\right)$ of (1). Imefim predicts a specific energy dependence which has a functional form different from traditional ones, cf. (13).

Before attempting the rather laborious derivation of the constants appearing in (13) from first principles, it has been tried to obtain some semiphenomenological results to find out whether the functional term of Imefim's energy dependence contradicts well established results or not. To that end two "short-cuts" have been taken:

(1) The amplitude of Imefim's potential/onesoliton slice, $\bar{U}_{0}\left(E_{\mathrm{p}}=0\right)$, has been normalized to phenomenological potentials.

(2) The constant $e$ appearing in (13) has for the time being been treated as an adjustable parameter.

However, in the course of time both phenomenological steps are to be removed by the further consistent development of Imefim. - The resulting agreement of Imefim's energy dependence with literature results based on formal grounds and sustained by the numerics, is a very nice one. In spite of the aforementioned phenomenological normalizations, we thus have the result that the energy dependence firmly predicted by Imefim is consistent with experience and conventional approaches. As discussed in Section 2.4. the latter holds in particular if one has in mind the notions of an energy dependent effective mass or of an energy dependent mass operator. The above findings certainly indicate that some of the features of Imefim lead to useful results/conclusions, yet, it requires a lot more work to remove the bits of phenomenology from Imefim and to understand how far this approach is to carry us.

\section{Acknowledgements}

E. F. H. would like to thank M. de Llano for facilitating his stay at the Instituto de Fisica of the UNAM, México, and he appreciates enlightning discussions with T. A. Brody, M. de Llano, E. S. Hernández and A. Mondragón. 
[1] K. A. Gridnev and E. F. Hefter, Phys. Letters 77 A, 490 (1980).

[2] E. F. Hefter, Nuovo Cim. 59 A, 275 (1980); Proc. XIV. Mikołajki Summer School on Nucl. Phys. (Mikołajki, Poland, Sept. 1981) Univ. of Warsaw, eds. B. Sikora and Z. Wilhelmi, 1982, p. 104; E. F. Hefter and K. A. Gridnev, ZfK-Rossendorf-Report (Rossendorf/Dresden, DDR) eds. G. Musiol, W. Wagner, and M. Josch 459, 128 (1981); V. M. Semjonov, K. A. Gridnev, E. F. Hefter, V. B. Subbotin, in "Some frontiers in nuclear physics" ed. E. F. Hefter (E. F. Hefter Hannover) ISBN 3-923471-00-9 (1982), p. 160.

[3] E. F. Hefter, Z. Physik C14, 87 (1982); Lett. Nuovo Cim. 32, 9 (1981).

[4] E. F. Hefter, Z. Naturforsch. 37 a, 1119 (1982).

[5] M. de Llano and E. F. Hefter, Contr. to the VI. Oaxtepec Nucl. Phys. Symposium, Jan. 1983 and in preparation.

[6] E. F. Hefter and I. A. Mitropolsky, LNPI-Preprint (LNPI, Gatchina/Leningrad district, USSR) 1983.

[7] E. F. Hefter, Prog. Theor. Phys. 69, 329 (1983).

[8] K. Chadan and P. C. Sabatier, Inverse Problems in Quantum Scattering Theory, Springer-Verlag, Berlin 1977.

[9] M. Bauer, E. Hernández-Saldaña, P. E. Hodgson, and J. Quintanilla, J. Phys. G: Nucl. Phys. 8, 525 (1982).

[10] G. Eder, H. Leeb, and H. Oberhummer, J. Phys. G: Nucl. Phys. 3, L127 (1977). - C. A. Engelbrecht and H. Fiedeldey, Ann. Phys. New York 42, 262 (1967).

[11] I. Kay and H. E. Moses, J. Appl. Phys. 27, 1503 (1956)

[12] C. S. Gardener, J. M. Greene, M. D. Kruskal, and R. M. Miura, Phys. Rev. Lett. 19, 1095 (1967); Comm. Pure Appl. Math. 27, 97 (1974); B. Fuchssteiner,
Commun. Math. Phys. 55, 187 (1977); M. Wadati and K. Sawada, J. Phys. Soc. Japan 48, 312 (1980).

[13] H. Grosse and A. Martin, Nucl. Phys. B 148, 413 (1979).

[14] J. F. Schonfeld, W. Kwong, J. L. Rosner, C. Quigg, and H. B. Thacker, Ann. Phys. New York 128, 1 (1980); I. Sabba-Stefanescu, J. Math. Phys. 23, 2190 (1982).

[15] A. C. Scott, F. Y. F. Chu, and D. W. McLaughlin, Proc. of the IEEE 61, 1443 (1973).

[16] K.-K. Kan and J. J. Griffin, Nucl. Phys. A301, 258 (1978); G. Gosh, B. Dutta-Roy, and M. Dey, J. Phys. G: Nucl. Phys. 3, 1077 (1977); D. S. Delion, K. A. Gridnev, E. F. Hefter, and V. M. Semjonov, J. Phys. G: Nucl. Phys. 4, 125 (1978); K. A. Gridnev, E. F. Hefter, K. Mikulas, V. M. Semjonov, and V. B. Subbotin, Aust. J. Phys. (in press).

[17] Y. Nogami and C. S. Warke, Phys. Rev. C17, 1905 (1978).

[18] E. H. Lieb and M. de Llano, J. Math. Phys. 19, 860 (1978); M. de Llano, Nucl. Phys. A317, 183 (1979).

[19] H. Reinhard, Phys. Lett. 121 B, 9 (1983).

[20] D. J. Kaup and A. C. Newell, Proc. Roy. Soc. London A361, 413 (1978); J. W. Miles, J. Fluid Mech. 91, 181 (1979).

[21] L. R. Rosen, J. C. Beery, and A. S. Goldhaber, Ann. Phys. New York 34, 96 (1965).

[22] S. T. Butler, R. G. L. Hewitt, B. H. J. McKellar, and R. M. May, Ann. Phys. New York 43, 282 (1967).

[23] H. R. Kidwai and J. R. Rook, Nucl. Phys. A 169, 417 (1971).

[24] W. T. H. van Oers, Phys. Rev. C3, 1550 (1971); R. M. Lerner, J. C. Hiebert, L. L. Rutledge Jr., and A. M. Bernstein, Phys. Rev. C3, 1254 (1972).

[25] G. Eckart and M. K. Weigel, J. Phys. G: Nucl. Phys. 2, 487 (1976). 